\title{
THE ROLE OF SIMULATION IN A STAGED LEARNING MODEL FOR NOVICE DRIVER SITUATIONAL AWARENESS TRAINING
}

\author{
Loren Staplin, Ph.D. \\ TransAnalytics, LLC \\ Kulpsville, Pennsylvania USA \\ E-mail: Lstaplin@transanalytics.com \\ James C. Dowdell \\ SafeDrive Technologies \\ Coraopolis, Pennsylvania USA \\ E-mail: Jdowdell@safedrivetech.com
}

\begin{abstract}
Summary: This paper theorizes that an optimal strategy for training novice drivers to acquire situational awareness skills will rely on a hierarchical approach consistent with traditional models of cognitive development. The success of applying such models hinges upon information presentation techniques that can maximize depth of processing, and hence comprehension and retention, at a specific stage of learning. Our general discussion argues that the appropriate use of simulation is uniquely suited to meet this need.
\end{abstract}

\section{INTRODUCTION}

The phenomenology of human learning and skill-building offers striking parallels between early cognitive development and the elaboration of mental models by adults. These parallels suggest that staged learning processes may also extend to novice driver training, as discussed in this paper. Conceiving of novice driver training as a staged learning process offers practical guidance in the optimization of training effectiveness, from the organization of program content to the requirements for tools and techniques best suited to its delivery to students. Our thesis is that, increasingly, the appropriate use of simulation will be a key discriminator of program success in reducing crashes by beginning drivers.

\section{BACKGROUND}

It is a widely cited statistic that motor vehicle crashes are the leading cause of death for people 15-20 years old (cf. NHTSA, 1999). The same sources document a sharp decline in motor vehicle-related deaths, controlled for exposure, beginning at age 20 and lasting until age-related functional deficits begin to significantly influence crash risk as drivers reach their 70's and 80's. The post-adolescent downturn in crash risk is presumed to be a consequence both of maturational processes which broadly affect decision making and risk taking behaviors, and of gains in the "situational awareness" of novice drivers through their early years' experience with varying road, weather, and traffic conditions.

Situational awareness is a term that has been used within the traffic safety community in different ways by different people. It is operationally defined in this paper as "visual search and allocation of attention resulting in vehicle control behaviors and safety outcomes consistent with expert 
(experienced) driver performance, in a specified operating context.” The operating contexts of particular interest here are those where an error in speed or space management by a driver is likely to result in a crash. These include demanding roadway geometries and operating conditions, and high traffic volumes, where there is exaggerated potential for conflict with other vehicles; plus degraded driving environments where the risk of single-vehicle, run-off-road crashes is magnified.

\section{GENERAL DISCUSSION}

The notion that prominent theories of human learning and cognitive development have special relevance to our thesis finds support in the work of researchers going back to Piaget (1962). This body of work holds that our minds, as active processors of information from our environment, develop structures or schemata as a function of experience, and that these structures in turn control our perceptions and understanding of the world. If the information or experience presented to a person "fits" with his/her mental structure, it will be understood and may be applied in ways that are adaptive for the individual. If it does not fit, the mind will reject it; and the mind must develop new structures or elaborate existing ones in order to accommodate the new information. These processes of assimilation (incorporating new experience into the mind's structures) and accommodation (changing a concept or forming a completely new concept) are at the heart of early childhood cognitive development and, interestingly, of many aspects of adult learning as well.

There is an orderly way in which schemata develop, as documented by Piaget and others who have made substantive contributions in this field (e.g., Bruner, 1973). Bruner's work is of particular interest because he has applied theories of the stages of cognitive development to education, with considerable influence on school curricula over the previous quarter century. Bruner, following Piaget, asserts that learning begins with an enactive stage, where direct interaction with the environment is crucial to the formation of new concepts. Physically orienting oneself in space, touching, grasping, learning the extent of one's reach and control through sensorimotor feedback to voluntary actions, is the first step. An iconic stage follows, characterized by the development and use of imagery. One key at this stage is the shift from a wholly egocentric world view to an ability to see the world from the perspective of others, including the visualization of one's own actions in a dynamic context where the actions of others are also represented. Then, at the most advanced stage of cognitive development, the symbolic stage, an individual learns to relate what is perceived in the immediate moment to an abstract representation of possible outcomes, extrapolating from present experience to construct a mental model of what might happen next - a critical element in risk perception.

Spatial information processing by adults, whereby an individual's exploration of a new environment leads to the construction of a "mental map" to guide navigation from an origin to a destination, provides an analogue of this sequence; i.e., an initial, egocentric cognitive model of the world is elaborated through experience into a representation of spatial relationships independent of one's own location or actions. This abstraction also has symbolic value to the driver, as affective qualities (road conditions, neighborhood characteristics, traffic density, etc.) distort cognitive distances and influence route choice (Staplin and Sadalla, 1981). 
These recurring patterns in human cognitive development suggest that the acquisition of safety concepts by novice drivers could be optimized through a training program that adheres to a staged learning model. This approach would present an ordered sequence of concepts to students, generally consistent with a progression from enactive, to iconic, to symbolic understanding of the driving task. During this progression through the program, students would only be asked to assimilate new information appropriate to their stage of learning.

Gaining an accurate sense of how one acts upon the world through control of a vehicle is a logical starting point. The vehicle is an extension of one's body, and the understanding of its extent, dynamics of motion, and orientation as a consequence of the driver's will are all learned through experience. Next, a "contextual” understanding of safety rules — specifically, the meaning of traffic regulations as they apply to the actual operations of driving, rather than facts learned "out of context" - reflects an associative process where devices and markings are related to road features and specific maneuvers. This depends strongly upon imagery, the student's ability to place him/herself in the situation. It also depends upon a shift from perceiving a driving situation strictly through one's own eyes; to an ability to visualize one's actions as perceived by others; to a complete understanding of the interactions of drivers and pedestrians at, for example, an intersection, as if viewed from above.

When a student's progression of training reflects this level of accommodation, an under-standing of the probabilities and risks of various behavioral choices in a situation becomes possible. This is an abstraction, to consider many possible consequences of one's actions and weigh decisions on the basis of both the likelihood and severity of a particular event. For example, the extent of drivers' braking and visual search behaviors at a passive railroad crossing where trains appear only rarely is revealing. Safe behaviors, if not partially reinforced through the occasional "near miss,” may be extinguished; and in the case of novice drivers, are not likely ever to be learned. Accurate perceptions of risk are essential to situational awareness, to guide a driver's visual search of the environment and to recognize and attend to cues which connote potential safety threats.

Providing the right training experience at the right time, to foster cognitive development resulting in situational awareness, is thus the challenge in training program development. And this is where appropriate simulation presents unique advantages as a complement to traditional classroom and behind-the-wheel techniques to enhance novice driver training.

It is not the intent here to exhaustively catalogue simulation techniques that may be of value as training aids; yet, certain tools and attributes appear best suited for use at particular stages of learning. It is suggested that the student's early understanding of vehicle orientation and guidance will benefit significantly from interactive driving simulation. There are hypothesized advantages in transfer of training as system fidelity increases, with full motion as the technology standard. However, system costs increase dramatically with added range and degrees of freedom for motion effectors, and cost-benefit relationships are unknown (Decina, Gish, Staplin, and Kirchner, 1996). With a focus on applications that are more practical for driver instruction on a large scale, systems which incorporate a (passenger) vehicle cockpit with conventional controls and displays, and which offer force feedback for steering control, imperceptible lag in system response to control inputs, and driving scene updates that are (subjectively) valid for brake and 
accelerator inputs should have a high training value during the early development of maneuvering skills. Information pertaining to conflict avoidance is not appropriate at this stage of learning, though, and content programming for the simulator should avoid dense traffic and high speeds in favor of less demanding scenarios.

Early stage learning addressing knowledge requirements for traffic regulations and the use of standard traffic control devices and roadway features can be enhanced through even more economical, desktop simulation tools. Specifically, computer-based training on a PC platform offers clear advantages over classroom methods in illustrating the context for applying instructions about how to use varying devices, road types and geometric features, thereby improving comprehension of lesson material. The iconic nature of this information also facilitates its retention and accessibility from working memory by the novice when behind the wheel.

As the novice enters a stage of learning where new concepts pertaining to protected, permitted, and prohibited vehicle movements must be internalized, involving dynamic relationships with other road users, the 3D and 4D visualization capabilities offered through desktop simulation become paramount. Supplementing lesson material with still images and with animation illustrates the perspectives of other drivers and gives a "bird's eye view" of a situation, and it can dramatize the cause-and-effect of specific driver (student) action or inaction as a situation unfolds.

When the student has developed an understanding of the driving task sufficient to assimilate information at the symbolic stage of learning, the value of interactive simulation arguably will be most pronounced. Abstract concepts of risk are operationalized through system capabilities that can elicit involuntary, visceral responses through realistic simulations of loss-of-control and collision events. These capabilities include - in addition to those noted above - a display resolution sufficient for discrimination of safety critical information at meaningful (apparent) decision sight distances, a (horizontal) field of view sufficient to encompass the priority safety threats in a given driving situation, and full collision dynamics for other vehicles and for natural and built features in the roadway environment. Program content for such simulation should include diverse weather, pavement, and visibility conditions, with complex traffic operations where sources of driver distraction and violations of expectancy can be precisely controlled. It is anticipated that the student's capabilities will be taxed during such simulations, and errors resulting in crashes are not only possible, but likely. The depth of processing associated with such events is intensified, and subsequent rehearsal and retrieval of safety lessons will be enhanced accordingly.

Finally, a simulation-enhanced curriculum improves standardization in presenting lesson materials and in scoring performance, while tailoring the pace of instruction and nature of feedback to the needs of individual students. These attributes are important not only to improve the feasibility of training driver situational awareness through a staged learning approach, but to insure that criteria for program graduation are applied fairly and to support subsequent evaluations of program validity. 


\section{References}

Bruner, J.S. (1973). Beyond the information given: Studies in the psychology of knowing. New York: Norton.

Decina, L.E., Gish, K.W., Staplin, L., and Kirchner, A.H. (1997). Feasibility of New Simulation Technology to Train Novice Drivers. U.S.DOT Pub. HS 808 548. Washington, DC.

National Highway Traffic Safety Administration, Traffic safety facts 1999: Young drivers (1999). U.S.DOT Pub. HS 809 099. Washington, D.C.

Piaget, J. (1962). “The stages of intellectual development of the child,” Bull. Menninger Clinic, 26(3).

Staplin, L. and Sadalla, E. (1981). “Distance cognition in urban environments,” Professional Geographer, 33(3). 